\title{
Author Correction: Resolving genetic heterogeneity in cancer
}

\section{Samra Turajlic, Andrea Sottoriva (D), Trevor Graham (D) and Charles Swanton (D)}

Nature Reviews Genetics (2019) https://doi.org/10.1038/s41576-019-0114-6 Published online 27 March 2019

The authors regret to report a minor error in Figure 2C. The location of peaks in the variant allele frequency (VAF) distribution was incorrectly drawn and labelled. The correct labels are as follows. The illustrated tumour has $50 \%$ tumour cells, and non-tumour cells are assumed to be strictly diploid. Clonal mutations on 1 of 4 copies of tumour DNA ( 1 in 6 molecules) are at VAF $=0.167$ (tetraploid loci, green), clonal mutations in 1 of 3 copies of tumour DNA ( 1 in 5 molecules) are at VAF=0.2 (triploid loci, orange), and clonal mutations in 1 of 2 copies of tumour DNA ( 1 in 4 molecules) are at $\mathrm{VAF}=0.25$ (diploid regions, blue). The neutral $1 / \mathrm{f}^{2}$-like tail is plotted in yellow. The lower detection threshold is drawn at $\mathrm{VAF}=0.03$. The revised figure below is correctly labelled. We note that this figure is an illustration only.

https://doi.org/10.1038/s41576-019-0188-1 I Published online 28 October 2019

Original

c Error propagation in clonal inference

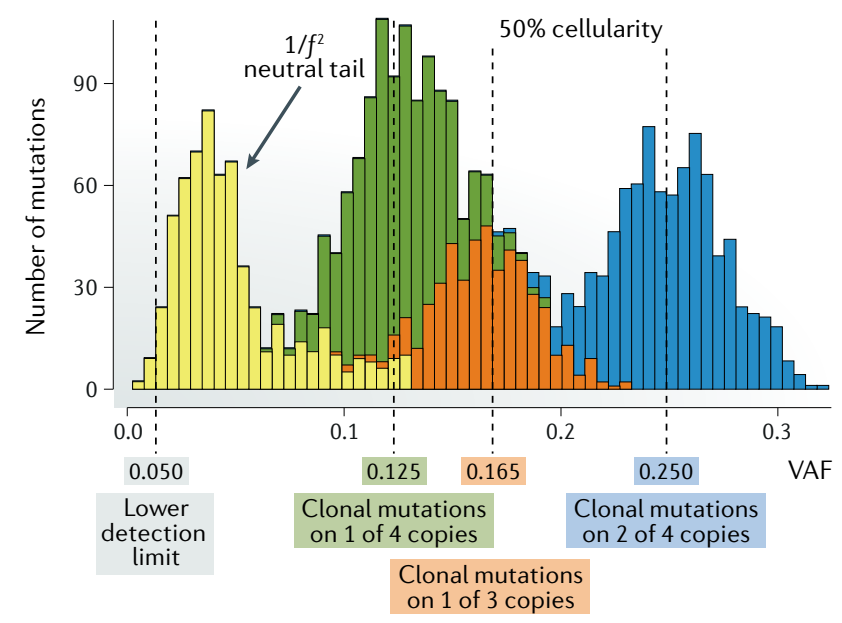

Fig. 2 | Original and Corrected.

\section{Corrected}

c Error propagation in clonal inference

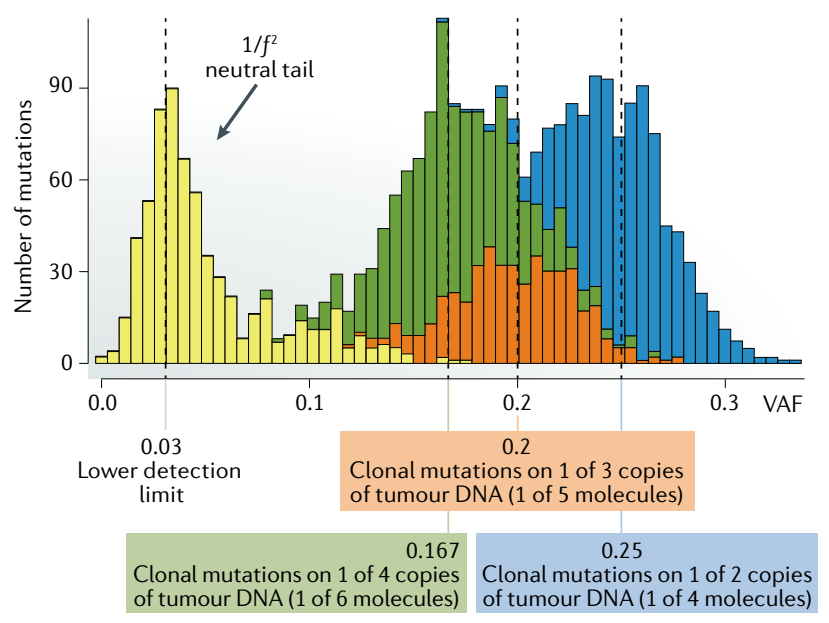

